\title{
Reading Emotional Faces \\ in Deaf and Hard-of-Hearing and Typically Hearing Children
}

Yung-Ting Tsou ${ }^{1 *}$, Boya $\mathrm{Li}^{1}$, Mariska E. Kret ${ }^{2,3}$, Inês Sabino da Costa ${ }^{1}$, Carolien Rieffe ${ }^{1,4}$

${ }^{1}$ Unit of Developmental and Educational Psychology, Institute of Psychology, Leiden University, Leiden, The Netherlands

${ }^{2}$ Cognitive Psychology Unit, Institute of Psychology, Leiden University, Leiden, The Netherlands

${ }^{3}$ Leiden Institute for Brain and Cognition (LIBC), Leiden, The Netherlands

${ }^{4}$ Department of Psychology and Human Development, Institute of Education, University College London, London, UK

* Correspondence concerning this article should be addressed to Yung-Ting Tsou, Unit of Developmental and Educational Psychology, Institute of Psychology, Leiden University, Pieter de la Court Building, Wassenaarseweg 52, 2333 AK, Leiden, The Netherlands, y.tsou@fsw.leidenuniv.nl.

To cite this paper: Tsou, Y.T., Li, B., Kret, M.E., da Costa, I.S., \& Rieffe, C. (in press).

Reading emotional faces in deaf and hard-of-hearing and typically hearing children. Emotion. https://doi.org/10.1037/emo0000863

(C)American Psychological Association, 2020. This paper is not the copy of record and may not exactly replicate the authoritative document published in the APA journal. Please do not copy or cite without author's permission. The final article is available, upon publication, at: https://doi.org/10.1037/emo0000863. 


\begin{abstract}
Reading emotions from other people's facial expressions is an important skill that guides social interactions. With limited auditory input and atypical emotion socialization, deaf and hard-of-hearing $(\mathrm{DHH})$ children may develop atypical processing patterns when reading emotional faces. The current study aimed at understanding whether and how DHH and typically-hearing $(\mathrm{TH})$ children differed at three emotion processing levels: gaze patterns, physiological arousal, and interpretation. Fifty-five DHH children and $72 \mathrm{TH}$ children completed an emotional face matching task in which they were presented with happy, angry, fearful, and emotionally-neutral faces. During the task participants' eye gazes and pupil diameter were measured by an eye-tracking device. The DHH and TH children both paid most attention to the eye region when reading emotional faces. Yet, a contrast between happy faces and non-happy faces was observed in physiological arousal and interpretation tendency in the DHH children only: Non-happy facial expressions were more arousing and were confused more often than happy expressions, which may reflect the DHH children being less experienced in processing non-happy expressions due to limited access to the social environment. The results highlighted the importance of looking into the qualitative differences between typical and atypical development.

Keywords: eye tracking; facial emotions; children; deaf and hard of hearing; psychosocial development.
\end{abstract}


Reading Emotional Faces in Deaf and Hard-of-Hearing and Typically Hearing Children Human facial expressions are important cues in daily life for interpreting the social environment and provide guidance during social interactions. This skill of "reading" or processing expressions of emotions in faces involves extracting, integrating, and interpreting information from facial features (Adolphs, 2002). This is achieved by children through the process of emotion socialization, when they interact with others, communicate their needs, observe social situations, or overhear conversations (Rieffe, Netten, Broekhof, \& Veiga, 2015; Saarni, 1999). However, these daily practices are less accessible to deaf and hard-of-hearing (DHH) children. The limited access to the social environment could hamper their development in other areas, and in turn further hinder their social participation. It is therefore important to examine how children process emotion expressions in faces during childhood, so that support may be provided early in life when needed. In this study we addressed this issue and focused on DHH and typical hearing $(\mathrm{TH})$ children's ability to process other people's facial emotion expressions at visual, physiological, and interpretation levels.

Many DHH children nowadays receive a hearing device, such as a hearing aid (HA) or a cochlear implant (CI). These technologies significantly improve their auditory performance and speech perception (Waltzman, 2006). Nevertheless, it is worth noting that hearing devices still have their limitations, such as a narrower hearing range and a lower ability to discriminate pitch information, which prevents users from perceiving subtle variations in daily conversations, especially in the presence of background noises or multiple speakers (Bacon, Opie, \& Montoya, 1998). Consequently, both before and after intervention, despite access to a hearing device, DHH children still have limited access to their social environment. This reduced access grants them fewer opportunities for emotion socialization, i.e., to learn emotion skills in their social context by participating in or observing social interactions, as compared to their TH peers (e.g., Calderon \& Greenberg, 2011; Rieffe et al., 2015).

This more difficult access to the social world impacts children's emotion socialization in different ways, including their ability to read people's facial emotion expressions. Previous studies showed more difficulties with matching different facial emotion expressions in DHH children compared to their TH peers at or before the age of 5 years (Wang, Su, Fang, \& Zhou, 2011; Wang, Su, \& Yan, 2016; Wiefferink, Rieffe, Ketelaar, De Raeve, \& Frijns, 2013). Yet, studies on older children showed comparable emotion-matching skills in DHH and TH children (Hosie, Gray, Russell, Scott, \& Hunter, 1998; Ziv, Most, \& Cohen, 2013), indicating that school-aged DHH children may catch up with their TH peers, at least regarding the mean level of accuracy.

To date, studies on reading facial emotion expressions in DHH children focused solely on the accuracy to recognize expressions. However, processing other people's facial emotions occurs at multiple levels in a parallel and coordinated manner, involving more aspects than the interpretation accuracy alone (Scherer, 2000). All these levels of processing can be informative. First, at the visual level, an observer fixates gazes on different facial features to extract information (Lundqvist \& Ohman, 2005). Second, at the physiological level, the observer experiences physiological arousal as an automatic aspect of the emotion-reading process. This enables them to immediately act upon an emotion-evoking situation (Frijda, 1986). Third, at the cognitive level, the observer interprets the observed facial expression by relating it to an emotion category as per past experiences (McClure, 2000). 


\section{Gaze Patterns in DHH Children}

Eye movements reveal how an individual scans and extracts information from the visual field. This is modulated by both top-down (goal-driven) and bottom-up (stimulusdriven) processes of attention (Liversedge \& Findlay, 2000). In typical development, signals with high relevance (goal-driven) and/or high salience (stimulus-driven) are fixated for longer than other sources of information. For example, emotional faces are looked at for longer than neutral faces (Bradley, Houbova, Miccoli, Costa, \& Lang, 2011); and eyes attract more attention than other facial regions (Schurgin et al., 2014). However, "relevance" and "saliency" are relational, depending on a person's prior experiences. Previous research has shown that with age, typically-developing children direct more attention towards the eye region than the mouth region (Meaux et al., 2014) and towards the inner features (i.e., eyes, nose, and mouth) than the other areas on emotional faces (Hunnius, de Wit, Vrins, \& von Hofsten, 2011; Naruse et al., 2013). Although infants exhibit an innate preference for human faces (Slater \& Quinn, 2001), their allocation of fixations over the inner features on the faces appears to develop with their increasing experiences in recognizing facial expressions (Hunnius et al., 2011).

Given an atypical hearing experience, DHH individuals may navigate the world in a way that differs from TH individuals to extract relevant information from faces. This was indeed what was found in the literature, despite the small body of studies. Letourneau and Mitchell (2011) found that American TH adults focused on the eye region when looking at emotional faces, while Deaf adults distributed their gazes more evenly on eyes and mouth, thus showing longer mouth-looking time than TH adults. Watanabe, Matsuda, Nishioka, and Namatame (2011) reported a main focus on the nose region in Japanese TH adults, whereas Deaf adults fixated longer on the eyes than the nose. These findings show that TH individuals tend to fixate on a core feature, which was the eye region in a Western population (Letourneau $\&$ Mitchell, 2011), and the nose region in an Eastern-Asian population (see Watanabe et al., 2011 for a cultural explanation of the tendency).

However, the patterns shown by DHH individuals was less clear from these two studies. A possible explanation for the reported patterns is that DHH individuals may tend to distribute their gazes to a greater area, thus showing longer fixations outside the core feature. This speculation is based on many previous studies that found DHH individuals to attend more to the peripheral visual field than TH individuals when detecting a shape on the screen (Bavelier et al., 2001; Proksch \& Bavelier, 2002). Possibly, congenital deafness leads to a preferred allocation of visual attention over the whole visual field to obtain more information, as a compensatory mechanism for the lack of auditory input (Dye, Hauser, \& Bavelier, 2008; Letourneau \& Mitchell, 2011; Proksch \& Bavelier, 2002).

It is worth mentioning that the studies above are all about DHH adults. To the best of our knowledge, only one study examined DHH children's visual attention towards emotional faces. Wang, Zhou, Cheng, and Bian (2017) found that DHH and TH children (aged 3 to 7 years) did not differ in gaze patterns on positive and neutral facial expressions, except for the condition with auditory cues. In this condition, DHH children looked shorter at the upper half of the face than TH children. These findings suggested that DHH children may perform differently from DHH adults during visual presentation of emotional faces. More data from children are needed in order to verify if this different gaze pattern for reading emotional faces 
emerges already in childhood.

\section{Physiological Arousal in DHH Children}

Physiological responses that cause pupil dilation, sweating, and an increased heart rate in the observer are part of the process of reading other people's emotions (Bradley, Miccoli, Escrig, \& Lang, 2008). This physiological arousal enables an observer to act immediately upon an emotion-provoking situation. In the recent decade, increasing research has been carried out examining pupillary responses to emotional stimuli in adults (e.g., Bradley et al., 2008; Laeng et al., 2013; Partala \& Surakka, 2003) and children (e.g., Geangu, Hauf, Bhardwaj, \& Bentz, 2011; Jessen, Altvater-Mackensen, \& Grossmann, 2016) because its noninvasive nature makes it a suitable measure for emotional arousal in various populations. A greater magnitude of pupil dilation reflects higher arousal (Bradley et al., 2008).

It has been consistently found that pupils dilate in response to both negative and positive emotional stimuli in adults (Bradley et al., 2008; Partala \& Surakka, 2003) and 6- to 12-month-old infants (Geangu et al., 2011). Moreover, in the studies that specifically used isolated emotional faces as stimuli, adults showed a greater pupil dilation towards negative facial expressions than positive ones (Laeng et al., 2013), while 7-month old infants demonstrated a reversed pattern (Jessen et al., 2016). This implies a possible developmental change that young children may experience greater arousal when facial expressions are most familiar and relevant in their daily social life (Campos et al., 2000; Jessen et al., 2016), whereas adults may develop a greater sensitivity to threatening signals which prepares them for a fight or flight (Kret \& de Gelder, 2013; Laeng et al., 2013).

Yet, the development of physiological reactivity may be affected by difficult parentchild interaction in early childhood. For example, children (aged 8 to 14 years) with a depressed mother were more strongly aroused by sad faces, while children with an anxious mother were more strongly aroused by angry faces, when compared to children whose mothers were not depressed or anxious, respectively (Burkhouse, Siegle, \& Gibb, 2014). Adolescents (aged 15 to 22 years) who thought their parents to be less socially supportive or communicatively skilled were more likely to exhibit higher arousal when talking about stressful events related to their parents (Afifi, Granger, Denes, Joseph, \& Aldeis, 2011). The higher arousal indicates higher sensitivity or more effort when handling negative emotions. Another study reported a reduced arousal level towards positive and negative emotion words in 18-year-old college students with depressed parents, reflecting decreased engagement in emotion-laden contexts (Bistricky, Ingram, Siegle, \& Short, 2014).

DHH children experience similar difficulties in parent-child interaction. Over $90 \%$ of the DHH children are born to TH parents (Mitchell \& Karchmer, 2004) and parent-child communication is often less effective in families with DHH children (Calderon \& Greenberg, 2011). However, it remains unexplored how DHH individuals physiologically respond to emotional information. To our knowledge, only two studies have investigated DHH children's emotional arousal using parent- or self-reports (Dirks et al., 2017; Netten et al., 2015). The studies indicated that DHH toddlers and adolescents did not differ from their TH peers in the parent- or self-rated levels of emotional contagion, i.e., the affective component of empathy that triggers personal arousal upon the witness of another person's emotion (Decety \& Jackson, 2004; Hoffman, 1987). Nevertheless, these outcomes did not necessarily reflect 
physiological responses.

\section{Interpretation Tendency in DHH Children}

The ability to understand the meaning of facial expressions is not innate. It requires an emotion socialization process in which children learn and practice the skill to relate a facial expression to their experiences. Children need to be exposed to social interactions to gain the experiences (Gordon, 1989; Rieffe et al., 2015). The quality and quantity of social interactions during the early years of life could thus affect how a person appraises a facial expression. That is, the interpretation of emotions is not definite but experiential. This view is supported by developmental research on the interpretation of emotion categories in TH children. Studies showed that TH children start with a valence-based interpretation of emotional faces (i.e., feeling good vs. feeling bad) and gradually develop the ability to differentiate same-valence facial expressions as discrete emotion categories (see Widen, 2013, for a review). As a result, young children are more likely to confuse between same-valence emotional faces (e.g., angry and fearful faces) than between opposite-valence emotional faces (e.g., happy and angry faces), whether verbal responses are required or not.

What DHH individuals "see" on an emotional face is a topic rarely analyzed. To our knowledge, Hosie et al. (1998) is the only study that reported the confusion pattern DHH children (aged 5-12.5 years) exhibited when recognizing emotional faces, which was similar to their TH peers: the younger children (5-8 years), whether DHH or TH, more often confused between the emotional faces within the negative valence, while the older children (8.5-12.5 years) mainly had difficulties with faces showing disgust. However, the picture emerges here is unclear because of the age difference between the younger $\mathrm{DHH}$ and TH children $\left(M_{\mathrm{DHH}}=\right.$ 6.6 years, $S D=1.3$ vs. $M_{\mathrm{TH}}=5.3$ years, $S D=0.3$ ).

In addition, prior studies that reported accuracy results also inform us DHH children's interpretation tendency. Using a matching task, Wiefferink et al. (2013) and Wang et al. (2016) found that DHH children (aged 2.5-7 years) had more difficulties differentiating between opposite-valence faces (e.g., happy and sad) and between same-valence faces (e.g., angry and sad) than their TH peers. When asked to point at an angry, fearful, happy, or neutral face among other faces, DHH children (2.5-7 years) more often chose an emotion expression not asked by the experimenter than their TH peers (Wang et al., 2011; Wang et al., 2016; Wiefferink et al., 2013). Moreover, DHH and TH children seem to differ in the emotion category they recognized best/worst. For example, Wiefferink et al. (2013) indicated that TH children were best at recognizing happy faces, yet DHH children (aged 2.5 to 5 years) performed similarly on all types of emotion expressions. Wang et al. (2011) showed that DHH children (aged 4 to 6.5 years) often chose an emotional face when asked for a neutral face, compromising their score on the neutral category, while TH children performed similarly on all expressions.

Taken together, these studies suggest that DHH and TH children may demonstrate different interpretation tendencies at least until middle childhood. More empirical evidence is needed to confirm the patterns DHH children exhibit when interpreting emotional faces.

\section{The Present Study}

DHH children have a different experience of emotion socialization due to hearing and 
communication difficulties that hinder their participation in social interactions. Past studies have shown that such an atypical childhood experience could influence the way DHH individuals respond to emotional signals, but studies on the underlying mechanisms and evidences from children are lacking, leaving a gap in the literature. Therefore, in this study we aimed to understand how DHH and TH children read other people's facial emotion expressions, considering three levels of facial emotion processing: gaze pattern, physiological arousal, and interpretation. We included children aged 3 to 10 years, since this is a period in which typically-developing children gradually learn and master the skill to identify different emotion categories (Durand, Gallay, Seigneuric, Robichon, \& Baudouin, 2007).

First, we examined DHH and TH children's gaze patterns while they were looking at emotional faces. We hypothesized that TH children would focus mostly on the core features (i.e., eyes or nose), while DHH children would tend to scan the entire face more evenly, thus looking longer at the mouth. We expected this pattern across the different emotion categories.

Second, we investigated the physiological arousal elicited by the observation of emotional faces through changes in pupil diameter. To the best of our knowledge, no research has been conducted to directly measure DHH individuals' physiological arousal towards emotional faces. Therefore, we based our hypothesis on the physiological arousal patterns found in children who had difficult emotion socialization in early childhood like DHH children (Afifi, Granger, Denes, Joseph, \& Aldeis, 2011; Bistricky, Ingram, Siegle, \& Short, 2014; Burkhouse, Siegle, \& Gibb, 2014). We expected a different pattern in physiological arousal, thus a different magnitude of pupil dilation, in the DHH group than in the TH group. We did not hypothesize on the direction of the difference given that hyper- and hypo-arousal were both observed in children with difficult emotion socialization experiences.

Third, we analyzed the interpretation children gave to the facial expressions to assess their interpretation accuracy and the tendency for recognizing faces as a certain emotion. Given the previous findings that school-aged DHH and TH children had comparable performances (Hosie et al., 1998; Ziv et al., 2013), we expected that DHH and TH children would identify facial expressions of basic emotions at a similar level of accuracy. Yet, we did not develop specific hypotheses for interpretation tendencies and group differences in these tendencies due to the lack of support.

Given that the present study aimed at understanding the underlying patterns DHH and TH children showed at the three processing levels, it is important that we considered not only the emotion categories predefined by the study but also the actual perceived emotion categories, which provides insight into how children actually "read" the faces (Russell \& Steinberg, 1994; Wagner, 1993). Therefore, all three levels of emotion processing were analyzed twice: once based on the predefined emotion category of the stimuli (i.e., the category intended by the design of the study), and once based on the interpreted emotion category (i.e., the emotion that the participant identified).

\section{Participants}

\section{Methods}

A total of 127 children in Taiwan aged between 3 and 10 years old participated in this study, including $55 \mathrm{DHH}$ children $\left(M_{\mathrm{age}}=6.45, S D_{\text {age }}=2.10\right)$ and $72 \mathrm{TH}$ children $\left(M_{\text {age }}=\right.$ $\left.6.03, S D_{\text {age }}=1.77\right)$. Children with additional disabilities were excluded from recruitment. 
Those who could not use oral language for communication were also excluded to narrow our focus on hearing status. All children and their parents were Asian. See Table 1 for participant characteristics.

Among the DHH children, five children wore HAs, and the other 50 children were implanted with a CI. All the DHH children had congenital or prelingual hearing loss. They received intervention (HA or $\mathrm{CI}$ ) before 2.5 years of age and had used the hearing device for a minimum duration of 7 months. As Table 1 shows, the DHH and TH children did not differ regarding their age, $t(125)=-1.22, p=.223$, gender distribution, $\chi^{2}(1, N=127)=.70, p$ $=.473$, nonverbal intelligence, $t(120)=1.77, p=.079$, or parental educational level, $t(118)=$ $1.45, p=.150$. Nonverbal intelligence was measured by two subscales of the Wechsler Preschool and Primary Scale of Intelligence Revised Edition (Block Design and Matrix subscales for children aged 3-5 years; D. Wechsler, 1989) or the Wechsler Intelligence Scale for Children Third Edition (Block Design and Picture Arrangement subscales for children aged 6-10 years; D. Wechsler, 1991).

This study was part of a research project that also includes other variables to examine social-emotional functioning in children with (a)typical development. Participants received a small gift (e.g., crayons) after finishing the experiments. All informed consent forms were signed by the primary caregivers on behalf of the child participants and by participants older than 7 years before the test procedures. The study protocol and informed consent form were approved by The Psychology Ethics Committee of Leiden University and Chang-Gung Memorial Hospital Ethics Committee for Human Studies.

Based on Wiefferink et al. (2013), a difference between DHH and TH children in their ability to discriminate basic emotions could be observed with a small-to-medium effect size ( $d$ $=.4$ ). Therefore, we estimated with a power analysis that a minimum sample size of 82 was required to be able to detect a group difference with an alpha of 0.05 and a power of $0.90 .{ }^{1} \mathrm{~A}$ total of 153 children were approached. After excluding children who did not finish at least $50 \%$ of the tasks that were part of the current study $(n=8)$, children who had additional disorders or low/high nonverbal intelligence ( $2 S D$ lower/higher or indicated by the teacher/responsible clinician; $n=17)$, and a child who accidentally took the test twice, the final sample included 127 children. The included $(n=127)$ and excluded $(n=26)$ samples did not differ in age, $t(151)=.62, p=.537$, gender distribution, $\chi^{2}(1, N=153)=2.40, p=.136$, and nonverbal intelligence, $t(142)=.08, p=.937$. Yet, the parents of the excluded sample had lower educational level than the parents of the included sample, $t(141)=2.42, p=.017$.

\section{Materials and Procedure}

Three levels of emotion processing were investigated, including gaze patterns, physiological arousal, and interpretation. The specific measurements at these levels are specified below. Children conducted an emotional match-to-sample task (Figure 1) in a quiet room at a cochlear implant center or at the participant's school, while their gazes and pupil size were being recorded by a Tobii X3-120 eye tracker (Tobii Technology, Sweden). A light meter was put in front of the computer screen and in front of the participant's eyes to control

\footnotetext{
${ }^{1}$ Note that originally we planned to use a mixed model ANOVA when estimating the sample size a priori and later changed to multilevel modeling considering the two-level structure of the data.
} 
for the illumination in the test environment. They were seated $65 \mathrm{~cm}$ away from the eye tracker mounted below a computer screen with a resolution of $1024 \times 768$ pixels. A 5-point calibration was conducted prior to each experiment. All participants were tested by the same experimenter, who received training on using the eye tracker before data collection.

Emotional match-to-sample task. Stimulus materials included angry, fearful, happy, and emotionally-neutral faces. There were 52 trials in total, counterbalanced by emotion category and face gender. All the faces were Asian, shown in a frontal view with direct gaze. A trial started with the presentation of a single facial expression, after which four faces of a different person were presented, one of which showing the same expression as before (Figure 1). Children were asked to choose the face expressing the same emotion as the one they saw at the start of the trial. Four practice trials were given prior to the start of the experiment. Only the children who understood the practice trials continued doing the testing trials.

Stimuli. Adobe Photoshop was used to standardize the photos so that all photos were grey scaled and in a vertical elliptical shape. Hair and impurities on the skin (e.g., moles, scars, and pocks) were removed. The average luminance in each photo was calculated and adjusted to the mean. The sample pictures were each resized to $480 \times 672$ pixels, and those used for recognition to $270 \times 378$ pixels. The faces were placed against a grey background which had the same mean luminance as the faces. Tobii Studio 3.4.8 was used for stimulus presentation. The face images $(n=620)$ were obtained from validated face databases upon the owners' approval (C. C. Chen, Cho, \& Tseng, 2013; L. F. Chen \& Yen, 2007; Lee, Kim, Yeon, Kim, \& Chae, 2013; Ma, Correll, \& Wittenbrink, 2015; Meuwissen, Anderson, \& Zelazo, 2017; Park et al., 2011; Tottenham, Borscheid, Ellertsen, Marcus, \& Nelson, 2002; Tottenham et al., 2009) and were randomly divided into four sets. Fifty-four typically-developing Asian adults (40 female, 13 male, 1 unreported) were given one of the sets and rated the faces regarding emotion category and intensity. The faces that were recognized above $70 \%$ correct were included in the experiment. Among the selected faces, those with the highest accuracy rate $(M=95.15 \%, S D=6.71)$ and highest intensity $(M=6.20, S D=1.09$, on a scale from 0 to $10)$ were chosen as sample stimuli, while the others were used in the recognition phase.

Gaze patterns. Gaze patterns were analyzed when participants watched the sample stimuli. The gaze patterns towards specific facial features were measured by calculating the ratio of total fixation duration within a predefined area of interest (AOI) against total fixation duration on the entire screen. Three AOIs were predefined: eyes, nose, and mouth (Figure 1). In addition, to examine the visual attention towards the faces, we calculated the ratio of total fixation duration on the entire face against the entire screen. The first $200 \mathrm{~ms}$ of each stimulation phase was discarded from calculation to reduce the lingering effect of the fixation cross (Kret, Roelofs, Stekelenburg, \& de Gelder, 2013).

Physiological arousal. The magnitude of arousal was measured by calculating the changes in pupil diameter from the baseline during the stimulation phase. Averaged pupil size during the final $500 \mathrm{~ms}$ of the fixation-cross presentation was used as the baseline for each trial. The first 2 seconds of the stimulation phase were discarded from analyses to avoid the influence from the picture-onset decrease in pupil size (Bradley et al., 2008). The signals during the last 2 seconds of the stimulation phase were down-sampled to $100-\mathrm{ms}$ timeslots for analysis. PhysioData Toolbox v0.2.2 (Sjak-Shie, 2019) was used to preprocess the raw pupil data. Following the guidelines suggested by Kret and Sjak-Shie (2019), we filtered out trial 
outliers (pupil size outside the feasible range, i.e., smaller than $1.5 \mathrm{~mm}$ or larger than $9 \mathrm{~mm}$; pupil size that changed more than 2 standard deviations of the dilation speeds of all pupil diameter samples in-between two adjacent data points), removed eye blinks, and interpolated gaps smaller than $250 \mathrm{~ms}$.

Interpretation accuracy and tendency. The answers the participants provided during the recognition phase were used for evaluating interpretation accuracy and tendency. Accuracy was regarded as the extent to which participants' answers agreed with the predefined emotion category. We used the unbiased hit rates (Hu scores) as an indicator of accuracy (Wagner, 1993). The $H u$ scores incorporated answers agreeing and disagreeing with the predefined category, allowing appropriate evaluation of accuracy especially in the cases that children constantly chose the same option throughout the trials. A simplified calculation example is given here: If there are two stimulus categories and two answer options in a forced-choice task (e.g., angry and neutral), the $H u$ score for the angry category is computed by the formula $\mathrm{A} /(\mathrm{A}+\mathrm{B}) * \mathrm{~A} /(\mathrm{A}+\mathrm{C})$, where $\mathrm{A}$ is the number of angry stimuli recognized as angry, $\mathrm{B}$ is the number of angry stimuli interpreted as neutral, and $\mathrm{C}$ is the number of neutral stimuli interpreted as angry. The $H u$ values range between 0 and 1 .

Further, we calculated the frequency that a certain type of answers was given to explore interpretation tendency. Using the same example above, we divided B by the total number of angry trials to obtain the frequency of angry faces misinterpreted as neutral.

Eye-tracking data processing. Via corneal reflection techniques, the Tobii X3-120 eye tracker recorded the position of participant's eyes and their diameter at a sampling rate of $120 \mathrm{~Hz}$. Tobii Studio 3.2.1 was utilized to process the data and the Tobii I-VT fixation filter was applied. AOIs were predefined on the software using its Dynamic AOI tool by drawing freeform shapes. Only the samples where both eyes were detected by the eye tracker (i.e., validation code was " 0 " for both eyes) were included in analyses.

\section{Statistical Analyses}

Statistical analyses were carried out using SPSS 25.0 (IBM Corp., Armonk, NY). Children with a CI and children with an HA were analyzed together as a group because leaving out children with an HA $(n=5)$ from the analyses did not change the direction of the results or the size of the effects (see Supplemental Materials 1 for the results after children with an HA were excluded). Group characteristics were compared using independent t-tests. Considering the wide age range in our participants, we added age as a covariate in all analyses. All independent variables were centered. Level of significance was set at $p<.05$.

Gaze patterns. Two generalized linear mixed models were used to analyze the gaze data considering the two-level data structure: trials (level 1) nested in participants (level 2). A final model was derived via a standard model selection procedure where nonsignificant factors were removed one by one from the full model, starting with higher-order interactions. Factors with a trend towards significance may still be included in the final model if removing them resulted in a worse model fit. A better model fit was indicated by a lower -2 log likelihood. A random intercept was included in both models. First, the fixation ratios within each of the AOIs were modeled to understand the gaze patterns towards specific facial features. Fixed effects included Age, Group (2: DHH, TH), Emotion (4: angry, fearful, happy, neutral), AOI (3: eyes, nose, and mouth), and interactions between Group and Emotion/AOI. 
Second, the fixation ratios within the entire face were modeled to examine participants' visual attention to the faces. Fixed effects include Age, Group, Emotion, and the interaction of Group x Emotion. Additionally, we developed extra models to analyze the fixation ratios based on the interpreted emotion categories (i.e., the emotion the participant identified). Here we excluded Emotion from the fixed effects and replaced it by Interpretation (4: interpreted as angry, fearful, happy, or neutral). Where post-hoc analyses were needed to interpret results, we repeated the models with the reference category changed (e.g., from neutral to happy emotion), or ran separate models in each group to confirm within-group effects (for such analyses, significance level was adjusted by the number of models, i.e., set at $p<\alpha / 2=.025$ ).

Physiological arousal. A generalized linear mixed model was developed to analyze the pupil data. A time factor at which the pupil diameter was sampled was added to the model of pupil diameter. Thus, the model had a nesting structure defined by repeated measures: time (level 1) nested in trials (level 2) nested in participants (level 3). To control for autocorrelation, a First Order Autoregressive (AR1) covariance structure was included at level 1 (i.e., time). A final model was derived following the same standard model selection procedure described previously. A random intercept was included. Fixed effects included Age, Group, Emotion, and the interaction of Group x Emotion. Linear, quadratic, and cubic terms were also added to model the changes in pupil size over time. An extra model for analyzing pupil size based on the interpreted emotion categories was developed, where Emotion was replaced by Interpretation as a fixed effect. Post-hoc analyses were conducted by repeating the models with the reference category changed, or by fitting separate models in each group (significance level set at $p<.025$ ).

Interpretation accuracy and tendency. $H u$ scores and frequency of misinterpretation were respectively analyzed by a multivariate analysis of variance. The $H u$ scores were analyzed with a 2 (Group) x 4 (Emotion) analysis of variance. When analyzing the frequency of misinterpretation, a 2 (Group) x 4 (Misinterpretation: misinterpreted as anger, fear, happiness, or neutral emotion) analysis of variance was conducted. To follow up significant within-group effects, pairwise $t$-tests with Bonferroni correction were applied by dividing $\alpha$ by the number of comparisons between the four emotion categories. Thus, adjusted significance level was set at $p<\alpha / 6=.008$. In both multivariate analyses Age was included as a covariate. Given that the DHH and TH groups did not differ in their mean age, it is legitimate to add Age as a covariate to analyses of variance to reduce the noise variance and improve the relationship between Group and the dependent variables (Miller \& Chapman, 2001). For exploratory purposes, we additionally used four 2 (Group) x 3 (Misinterpretation: e.g., anger misinterpreted as fear, happiness, or neutral emotion) multivariate analyses of variance to examine how each predefined emotion category was confused with the other emotion categories. More details and results of these exploratory analyses can be found in Supplemental Materials 2.

Effect size. For generalized linear mixed models, we report standardized effect sizes $(\delta)$ calculated using the formula provided by Raudenbush and Liu (2000), which is an extension to Cohen (1988) approach suitable for a multilevel design. Raudenbush and Liu (2000) suggested that a $\delta$ of $.20, .50$, and .80 respectively indicates a small, medium, and large effect size. For multivariate analyses of variance, we used partial eta squared $\left(\eta_{p}^{2}\right)$ for effect size comparison with $.01, .06$, and .14 being respectively viewed as a small, medium, and 
large effect size (Cohen, 1988).

Missing data. Of one DHH child and three TH children, eye tracker data could not be acquired due to device failure. Nonverbal intelligence scores were missing from four DHH children and one TH child. Five parents of the DHH children and two parents of the TH children did not report their educational level. Little's MCAR test (Little, 1988) showed that the data were missing completely at random, $\chi^{2}=3997.08, d f=3990, p=.465$.

\section{Results}

\section{Gaze Patterns}

First, we analyzed the gaze data within each AOI as a function of the predefined emotion categories. We found main effects of Emotion, $F(3,15177)=2.70, p=.044$, and AOI, $F(2,15177)=2678.50, p<.001$ (Table 2 and Figure $2 \mathrm{~A})$. All children looked longer at the AOIs in fearful faces than the other emotional faces, $b=.01, t=2.04, p=.042,95 \% C I$ $[.00, .02], \delta=.05$, and longer at the eyes than the mouth, $b=.27, \mathrm{t}=55.45, p<.001,95 \% C I$ $[.26, .28], \delta=1.08$, which was looked at longer than the nose, $b=-.07, t=-13.65, p<.001$, $95 \% C I[-.08,-.06], \delta=.27$. No effects related to Age or Group were observed.

Second, we modeled the gaze data within the entire face as a function of the predefined emotion categories. We found a main effect of Emotion, $F(3,5057)=3.84, p$ $=.009$ (Table 2). In all children, angry and fearful faces were looked at longer than neutral faces, i.e., the reference category (angry: $b=.012, t=2.67, p=.008,95 \% C I[.003, .02], \delta$ $=.10$; fearful: $b=.014, t=3.16, p=.002,95 \% C I[.01, .02], \delta=.12)$, while happy faces were looked at equally as neutral faces. No effects related to Age or Group were observed.

In order to rule out group differences in gaze behavior on the basis of how the children interpreted the emotions, we replaced the factor Emotion with Interpretation and ran the two above models again. As in the first model, the third model showed a main effect of AOI, $F(2$, $15180)=2677.59, p<.001$ (Table 2). Fourth, when investigating the fixation ratios within the entire face, none of the effects reached significance (Table 2). No effects related to Age, Group, or Interpretation were observed.

\section{Physiological Arousal}

Analyzing the pupil data based on the predefined emotion category, we found an interaction of Group $x$ Emotion, $F(3,55074)=3.28, p=.020$ (Table 2 and Figure 2B). To interpret this interaction, we first repeated the model with each predefined emotion category as the reference category. In all these repeated models, the main effect of Group was not observed, indicating that the DHH group did not differ from the TH group in their arousal towards the referenced emotions. Next, we conducted separate models in each group to confirm the within-group effect of Emotion (significance level set at $p<\alpha / 2=.025$ ). Respectively using each predefined emotion category as the reference, we found that the DHH children were more aroused by angry and neutral faces than by happy faces (anger faces: $b=.07, t=2.63, p=.009,95 \% C I[.02, .11], \delta=.17$; neutral faces: $b=.06, t=2.33, p$ $=.020,95 \% C I[.01, .11], \delta=.15)$, and there was no difference between fearful and happy faces, nor between the three non-happy emotions. The TH children were similarly aroused by the four types of faces (see Supplemental Materials 3 for these post-hoc analyses). No other effects involving Age, Group, and Emotion were observed. Moreover, when the pupil data 
were analyzed based on the interpreted emotion categories, none of the fixed effects were significant (Table 2).

\section{Interpretation Accuracy and Tendency}

Using the unbiased $H u$ scores as the dependent variable, we found a main effect of Emotion, $F(3,372)=64.42, p<.001, \eta_{p}^{2}=.34$ (Figure 2C). No effects involving Group were observed. Pairwise comparisons showed that all children recognized happy faces better than neutral faces, $t(125)=8.32, p<.001,95 \% C I[.10, .16]$, which were interpreted better than angry and fearful faces, $t \mathrm{~s}>5.11, p \mathrm{~s}<.001$. No difference was found between angry and fearful faces, $t(126)=.28, p=.784,95 \% C I[-.03, .04]$. An older Age was related to a higher $H u$ score, $F(1,123)=67.99, b=.005, p<.001,95 \% C I[.004, .007], \eta_{p}^{2}=.36$.

Next, the frequency of misinterpretation was used as the dependent variable to examine which emotion category was most frequently chosen when it was not the target. We found a main effect of Misinterpretation, $F(3,372)=15.62, p<.001, \eta_{p}^{2}=.11$, and an interaction of Group x Misinterpretation, $F(3,372)=7.77, p<.001, \eta_{p}^{2}=.06$ (Figure 2D). Post-hoc pairwise comparisons showed that the DHH children more often chose an angry face than the TH children, $t(125)=2.08, p=.039,95 \% C I[.001, .07]$, while the TH children more often chose a neutral face than the DHH children, $t(124)=-3.32, p=.001,95 \% C I[-.06$, -.02]. Also, the DHH children more often chose angry faces than fearful faces, $t(54)=2.86, p$ $=.006,95 \% C I[.01, .07]$, which were chosen similarly often as neutral faces, $t(54)=1.29, p$ $=.204,95 \% C I[-.01, .04]$, but more often than happy faces, $t(54)=3.50, p=.001,95 \% C I$ $[.01, .05]$. The TH children more often chose angry and neutral faces over fearful and happy faces, $t \mathrm{~s}>3.14, p<.003$, while there was no difference between fearful and happy faces, $t(71)$ $=.57, p=.569,95 \% C I[-.01, .02]$. An older Age was related to a lower frequency of misinterpretation, $F(1,123)=42.84, b=-.001, p<.001,95 \% C I[-.001,-.001], \eta_{p}^{2}=.26$.

\section{Discussion}

Children learn to process emotions when they participate in or observe others during social interactions, and this skill in turn becomes one of the fundamental elements in their interactions with others. It is therefore crucial to study emotion processing in children who have less access to the social world, such as DHH children, to understand the effect of atypical emotion socialization on the development of this skill. The present study examined how 3- to 10-year-old DHH and TH children read static emotional faces at three underlying processing levels: gaze patterns, physiological arousal, and interpretation. We found that the DHH and TH children developed a similar level of accuracy in reading faces expressing basic emotions and showed a similar gaze pattern that focused more on the eyes and less on nose and mouth. Yet, dissimilarities were also found between the two groups at physiological and interpretation levels. While the TH children found all facial expressions similarly arousing and more often incorrectly chose angry or neutral faces over fearful and happy faces, the DHH children were more strongly aroused by angry and neutral faces than by happy faces, and more often incorrectly chose non-happy (angry, fearful, and neutral) faces over happy faces. Below we will discuss these outcomes and their implications.

The hypothesis of different gaze patterns towards facial features between DHH and 
TH children was not supported by our findings. This outcome may be related to the context in which facial cues are used by DHH individuals. In the studies by Letourneau and Mitchell (2011) and Watanabe et al. (2011), Deaf adults, who used sign language as their main mode of communication, distributed their gazes to a greater area of the emotional faces than TH adults, which seems relevant when using sign language. In the study by Wang et al. (2017), children were tested with and without verbal cues, and DHH children looked shorter at the upper part of the emotional faces than their TH peers, but only in the condition with verbal cues. Very likely, DHH children still partly rely on lipreading as their hearing is not on the level of their TH peers despite a CI or HA. Taken together, these past studies show differential attention on facial features between DHH and TH individuals, when (verbal or sign) language is involved. However, in the current study, all stimuli were presented only visually and none of the children used sign language. In such a context, the facial areas that are most relevant to emotion recognition, i.e., the eyes in our case, attracted more attention of DHH and TH children. Our finding, together with the previous studies on DHH participants, suggests that DHH individuals use facial cues adaptively according to the situation.

Despite the similar level of accuracy and similar gaze pattern towards facial features in the DHH and TH children, the DHH children exhibited a contrast between happy and nonhappy facial expressions in physiological arousal and interpretation tendency that was not observed in the TH children. These findings are partly expected, although we based our hypotheses on studies that involved other clinical groups than the one we tested (Afifi, Granger, Denes, Joseph, \& Aldeis, 2011; Bistricky, Ingram, Siegle, \& Short, 2014; Burkhouse, Siegle, \& Gibb, 2014). Similar to those studies, our results show that children with atypical emotion socialization experiences may exhibit characteristic arousal and interpretation patterns when processing emotions. There could be two potential explanations for this happy vs. non-happy contrast in DHH children. First, the contrast may be considered threat sensitivity: DHH children may be more vigilant towards stimuli they consider negative and threatening in order to be prepared for a "fight or flight" (Field \& Lester, 2010; Kret \& de Gelder, 2013; Laeng et al., 2013). However, this hypothesis was not fully supported by our results. If the threat-sensitive state were what we observed in the DHH children, the DHH children were supposed to show higher arousal towards negative emotions than the $\mathrm{TH}$ children, and group differences should have been more pronounced when the interpreted emotion category was taken into the analysis, which were not the case in the current study.

The second "familiarity" hypothesis may be a more possible explanation: DHH children may be less familiar with non-positive emotions because of an (over-)protective environment provided by their parents (Calderon \& Greenberg, 2011; Holmbeck et al., 2002). Given the hardship DHH children experienced in their childhood due to medical examinations, surgery, and rehabilitation, their parents are often reported to show overprotection (Calderon \& Greenberg, 2011; Pinquart, 2013). A highly protective family setting, along with limited access to the social environment, could result in reduced exposure to emotions that are not positive, which requires more cognitive resources to process. In this study, the DHH children were more physiological aroused by angry and neutral faces than by happy faces, while no differences were found between angry/fearful faces and neutral faces; also, the DHH children more often incorrectly chose an angry, fearful, or neutral faces over happy faces. These results together suggested that the DHH children may devote more effort 
to process the emotional faces less familiar to them yet still experience more confusion between these faces. This may have implications for rehabilitative trainings as the exposure to different emotions appears to be of importance.

\section{Limitations and Future Directions}

This current study had the strength of investigating underlying processing mechanisms of emotion recognition in DHH children. This topic was hardly studied in the past. It was partly because DHH children who had better spoken language or milder hearing loss were often considered to have better social adjustment, and thus more focus was on the hearing and language factors rather than socioemotional development. However, during the past decade an increasing number of studies has shown that children with a CI or HA still exhibited difficulties in developing various social-emotional skills despite their stable hearing and language abilities (Netten et al., 2015; Rieffe et al., 2018). This suggests that limited access to the social environment could hinder social learning, and that more studies are needed to understand its impact. The advanced eye-tracking technology developed in recent years also made it possible to measure young children's eye movement and pupil size in a reliable and unobtrusive manner. Therefore, the current study took a step forwards by examining the mechanisms involved in emotion recognition. Our findings underline the importance of emotion socialization, which could be associated with atypical physiological and cognitive processing of emotions. However, several limitations of this study should be taken into account when interpreting the results.

First, this study had a cross-sectional design and included a wide age range. Although we controlled for age in all analyses, further investigations are needed in order to better account for the following two findings. The first one is in regard to the age effect found only in children's interpretation but not in their gaze pattern and physiological arousal. This indicates that the gaze/arousal patterns observed in the present study might already be observed during toddlerhood, which remain relatively stable across time at least until 10 years of age. The finding that children improve their interpretation with age may imply that they become more and more proficient in using the processing patterns they developed. Yet, no conclusion may be drawn without longitudinal data. The second finding that cannot be well explained by the current design is the relation between gaze/arousal patterns and interpretation tendency. Given a lack of experimental manipulation on the timeline, we could not conclude on the potential (causal) link between these parallel processing levels. However, past studies have shown both top-down and bottom-up influences: Attention and physiological arousal contribute to the appraisal of emotional signals (Gray, Harrison, Wiens, \& Critchley, 2007; White, Suway, Pine, Bar-Haim, \& Fox, 2011), whereas a pre-existing interpretation tendency may affect arousal pattern (Joormann, Waugh, \& Gotlib, 2015) and direct attention towards cues inconsistent with expectation (Horsley, de Castro, \& Van der Schoot, 2010).

Second, in this study we ran separate analyses using predefined and interpreted emotion categories as independent variables respectively, and found an interaction for group and emotion categories predicting physiological arousal only when we included the predefined categories, but not the interpreted categories. When we analyzed the predefined categories, we considered the responses elicited by viewing the emotional expressions 
(without necessarily consciously interpreting them). When the interpreted categories were included in the analyses, participants' decision-making process was also taken into account. Previous studies have shown that people may react to an emotional expression differently depending on whether they interpret it as a threat. For example, socially anxious people may regard smiles as threatening and thus show stronger physiological responses than non-anxious individuals towards these stimuli (e.g., Garner et al., 2011). Therefore, conducting the analyses with predefined and interpreted emotion categories may help us disentangle the viewing process from the decision-making process that were involved in our study. Yet, given the exploratory nature of such analyses and the lack of previous literature, we can only provide speculative explanations to any discrepancy observed between the two analyses. Our finding that the group interaction in physiological arousal was only observed for predefined emotion categories might suggest that the group difference lay in the viewing process, rather than in the decision-making process. Perhaps, the presentation of angry and neutral expressions caused more confusion, and thus higher levels of arousal, in the DHH children than happy expressions because DHH children have likely had less experience with these expressions of emotion. Moreover, different brain mechanisms might be involved. For people to interpret the emotional expressions of others, the recruitment of social brain areas informing decision-making brain areas is required. For viewing the emotional expressions, the process relies largely on the lower-level subcortical networks (Adolphs, 2002, 2006). Perhaps, DHH children's cortical network was over-active when viewing emotional faces because they needed to put more effort into identifying the emotions, which overshadowed the lower-level subcortical effects (see Kret, Denollet, Grèzes, \& de Gelder, 2011, for a similarly overactivated cortical network in an adult population with social inhibition). Although viewing and interpreting emotions were obviously not completely independent, they did capture slightly different variance across the two populations. This discrepancy might reflect group differences in the underlying mechanisms, which requires further investigations using multidimensional measures.

Third, some limitations regarding the stimuli used in the current study need to be considered. We examined only static faces showing highly expressive basic emotions posed by actors/actresses. In real life, emotion processing usually involves subtle, spontaneous expressions and more complex, or even mixed, emotions, which are expressed through different modalities (e.g., face, voice, and body) and need to be evaluated together with the social context (Scherer, 2000). To increase ecological validity, more dimensions could be considered in future studies. For this reason, the current finding that DHH and TH children showed similar levels of accuracy and gaze patterns should be interpreted with caution. When the emotion to be processed involves more dimensions, children may experience more difficulties. Very likely, the unfamiliarity towards non-happy signals that we observed in the processing of basic emotions may also be found in DHH children's understanding of more complex emotional events.

Moreover, the current study used only adult faces as stimuli. Although in adults an own-age advantage has been found in face processing (see Rhodes \& Anastasi, 2012 for a review), developmental studies showed that children (aged 3 to 16 years) better recognized adult faces than child faces, possibly because they have accumulated more experiences interacting with adults since birth, and adult faces carry more relevant, determinant 
information for, e.g., rewards and punishment (Hoehl, Brauer, Brasse, Striano, \& Friederici, 2010; Macchi Cassia, Pisacane, \& Gava, 2012; Marusak, Carre, \& Thomason, 2013). Considering together with the fact that validated face sets with Asian child faces are very limitedly available, we included only adult faces in this study. Yet, we also suggest future studies to use peer faces, given the neural responses induced by adult and child faces were found to be different in recent studies (Hoehl et al., 2010; Marusak et al., 2013). It is therefore crucial that validated face sets including non-Western child faces can be developed.

Fourth, some considerations are also required regarding the background of participants. DHH refers to a very heterogeneous population. Yet, the majority of the DHH children in this study had a CI, meaning that most of them had severe-to-profound hearing loss (i.e., hearing threshold $>70 \mathrm{~dB}$ HL). Currently, we could only conclude that excluding the children with HAs did not change the results. Future studies may take into account the heterogeneity in the DHH population to further increase our knowledge on the association between auditory input and emotion socialization. In addition, Taiwanese children were recruited in the current study. Prior studies have shown that Western and East Asian people use different gaze patterns for reading emotional faces: Eastern Asian viewers fixated at the eye region for a longer time than Western viewers (Jack, Blais, Scheepers, Schyns, \& Caldara, 2009; Jack, Garrod, Yu, Caldara, \& Schyns, 2012). This may also explain why our study found both DHH and TH groups to focus on the eye region, which appears to be an important cue for emotion recognition in East Asians. Likewise, Westerners and East Asians differed in the interpretation of emotion category (e.g., Dailey et al., 2010) and in how physiological arousal was moderated (e.g., Chentsova-Dutton, Tsai, \& Gotlib, 2010). Therefore, future studies are needed to understand the generalizability of the results in this study.

Importantly, the ability to recognize emotion expressions is often found to predict internalizing and externalizing behaviors, such as depression, anxiety, hyperactivity, and aggression (Chronaki et al., 2015; Trentacosta \& Fine, 2010). Past research did show a higher prevalence (about twofold) of these psychopathological symptoms in DHH children than in TH children (Stevenson, Kreppner, Pimperton, Worsfold, \& Kennedy, 2015). Family support, parent-child interaction, and communicative skills were found to be important factors contributing to fewer internalizing and externalizing behaviors (Theunissen et al., 2015; van Eldik, Treffers, Veerman, \& Verhulst, 2004), whereas the degree of hearing loss and mode of communication were not (Dammeyer, 2010; Fellinger, Holzinger, Sattel, \& Laucht, 2008). The results in general support the view that limited access to social environment, rather than the deafness itself, is a barrier that hinders the development of children's social competence. Here, our findings on the underlying processing of emotional information provide a new perspective that may help improve the knowledge on the causes underlying the emotionalbehavioral difficulties in DHH children. Future research is required to investigate this link by including measures for psychopathological symptoms, such as depression and aggression, and interpersonal relations, such as peer acceptance and rejection.

\section{Conclusions}

To conclude, the current study calls for the need to look beyond accuracy and into the possible qualitative differences between children with typical and atypical emotion socialization experiences. The DHH and TH children in this study did not differ in their 
accuracy and gaze patterns when recognizing facial emotions, yet the two groups of children differed in physiological arousal patterns and interpretation tendencies when processing nonpositive emotions in faces. Such differences reflect that the DHH children may devote more effort to processing the emotional faces less familiar to them. The results highlight the benefit of measuring accuracy along with other dimensions that help us gain a deeper insight into children's behaviors. Moreover, our findings underlie the importance of social access for DHH children to acquire sufficient emotional knowledge. Importantly, what we found in DHH children may also be relevant to other clinical groups who experience similar difficulties in social interactions, such as children with Developmental Language Disorder (DLD) and children with Autism Spectrum Disorder (ASD). These children, though not the majority, take up about $9 \%$ of world's pediatric population $(1.5 \%$ for $\mathrm{DHH}$; $7 \%$ for DLD; $0.6 \%$ for ASD; Norbury et al., 2016; Tomblin et al., 1997; WHO, 2018, 2019). Atypical outcomes found in these children are not necessarily impairments but signals that indicate different learning experiences and the need for a more accessible social environment for easier acquisition of social-emotional knowledge. 
Reference

Adolphs, R. (2002). Recognizing emotion from facial expressions: psychological and neurological mechanisms. Behavioral and Cognitive Neuroscience Reviews, 1(1), 21 62.

Adolphs, R. (2006). Perception and emotion: How we recognize facial expressions. Current Directions in Psychological Science, 15(5), 222-226.

Afifi, T. D., Granger, D. A., Denes, A., Joseph, A., \& Aldeis, D. (2011). Parents communication skills and adolescents salivary $\alpha$-amylase and cortisol response patterns. Communication Monographs, 78(3), 273-295.

Bacon, S. P., Opie, J. M., \& Montoya, D. Y. (1998). The effects of hearing loss and noise masking on the masking release for speech in temporally complex backgrounds. Journal of Speech, Language, and Hearing Research, 41(3), 549-563.

Bavelier, D., Brozinsky, C., Tomann, A., Mitchell, T., Neville, H., \& Liu, G. (2001). Impact of early deafness and early exposure to sign language on the cerebral organization for motion processing. The Journal of Neuroscience, 21(22), 8931-8942.

Bradley, M. M., Houbova, P., Miccoli, L., Costa, V. D., \& Lang, P. J. (2011). Scan patterns when viewing natural scenes: Emotion, complexity, and repetition. Psychophysiology, 48(11), 1544-1553.

Bradley, M. M., Miccoli, L., Escrig, M. A., \& Lang, P. J. (2008). The pupil as a measure of emotional arousal and autonomic activation. Psychophysiology, 45(4), 602-607.

Bistricky, S. L., Ingram, R. E., Siegle, G. J., \& Short, M. (2014). Parental depression risk and reduced physiological responses during a valence identification task. Cognitive Therapy and Research, 39(3), 318-331.

Burkhouse, K. L., Siegle, G. J., \& Gibb, B. E. (2014). Pupillary reactivity to emotional stimuli in children of depressed and anxious mothers. Journal of Child Psychology and Psychiatry, 55(9), 1009-1016.

Calderon, R., \& Greenberg, M. T. (2011). Social and emotional development of Deaf children: family, school, and program effects. In M. Marschark \& P. E. Spencer (Eds.), Oxford Handbook of Deaf Studies, Language, and Education (2nd ed., Vol. 1, pp. 188-199). New York, NY: Oxford University Press.

Campos, J. J., Anderson, D. I., Barbu-Roth, M. A., Hubbard, E. M., Hertenstein, M. J., \& Witherington, D. (2000). Travel broadens the mind. Infancy, 1(2), 149-219.

Chen, C. C., Cho, S. L., \& Tseng, R. Y. (2013). Taiwan Corpora of Chinese Emotions and Relevant Psychophysiological Data: Behavioral evaluation norm for facial expressions of professional performer. Chinese Journal of Psychology, 55, 439-454.

Chen, L. F., \& Yen, Y. S. (2007). Taiwanese Facial Expression Image Database. National Yang-Ming University, Taipei, Taiwan.

Chentsova-Dutton, Y. E., Tsai, J. L., \& Gotlib, I. H. (2010). Further evidence for the cultural norm hypothesis: positive emotion in depressed and control European American and Asian American women. Cultural Diversity \& Ethnic Minority Psychology, 16(2), 284-295.

Chronaki, G., Garner, M., Hadwin, J. A., Thompson, M. J., Chin, C. Y., \& Sonuga-Barke, E. J. (2015). Emotion-recognition abilities and behavior problem dimensions in preschoolers: evidence for a specific role for childhood hyperactivity. Child Neuropsychology, 21(1), 25-40.

Cohen, J. (1988). Statistical Power Analysis for the Behavioral Sciences (2nd ed.). Hillsdale, NJ: Lawrence Erlbaum Associates.

Dailey, M. N., Joyce, C., Lyons, M. J., Kamachi, M., Ishi, H., Gyoba, J., \& Cottrell, G. W. (2010). Evidence and a computational explanation of cultural differences in facial expression recognition. Emotion, 10(6), 874-893. 
Dammeyer, J. (2010). Psychosocial development in a Danish population of children with cochlear implants and deaf and hard-of-hearing children. Journal of Deaf Studies and Deaf Education, 15(1), 50-58.

Decety, J., \& Jackson, P. L. (2004). The functional architecture of human empathy. Behavioral and Cognitive Neuroscience Reviews, 3(2), 71-100.

Dirks, E., Ketelaar, L., van der Zee, R., Netten, A. P., Frijns, J. H. M., \& Rieffe, C. (2017). Concern for others: a study on empathy in toddlers with moderate hearing loss. Journal of Deaf Studies and Deaf Education, 22(2), 178-186.

Durand, K., Gallay, M., Seigneuric, A., Robichon, F., \& Baudouin, J.-Y. (2007). The development of facial emotion recognition: The role of configural information. Journal of Experimental Child Psychology, 97(1), 14-27.

Dye, M. W. G., Hauser, P. C., \& Bavelier, D. (2008). Visual attention in Deaf children and adults: implications for learning environments. In M. Marschark \& P. C. Hauser (Eds.), Deaf Cognition: Foundations and Outcomes (pp. 250-263). New York, NY: Oxford University Press.

Fellinger, J., Holzinger, D., Sattel, H., \& Laucht, M. (2008). Mental health and quality of life in deaf pupils. European Child and Adolescent Psychiatry, 17(7), 414-423.

Field, A. P., \& Lester, K. J. (2010). Is there room for 'development' in developmental models of information processing biases to threat in children and adolescents? Clinical Child and Family Psychology Review, 13(4), 315-332.

Frijda, N. H. (1986). The emotions. Paris, France: Editions de la Maison des Sciences de l'Homme.

Garner, M., Clarke, G., Graystone, H., \& Baldwin, D. S. (2011). Defensive startle response to emotional social cues in social anxiety. Psychiatry Research, 186(1), 150-152.

Geangu, E., Hauf, P., Bhardwaj, R., \& Bentz, W. (2011). Infant pupil diameter changes in response to others' positive and negative emotions. PLoS ONE, 6(11), e27132.

Gordon, S. L. (1989). The socialization of children's emotions: Emotional culture, competence, and exposure. In C. Saarni \& P. L. Harris (Eds.), Cambridge Studies in Social and Emotional Development. Children's Understanding of Emotion. (pp. 319349). New York, NY: Cambridge University Press.

Gray, M. A., Harrison, N. A., Wiens, S., \& Critchley, H. D. (2007). Modulation of emotional appraisal by false physiological feedback during fMRI. PLoS ONE, 2(6), e546.

Hoehl, S., Brauer, J., Brasse, G., Striano, T., \& Friederici, A. D. (2010). Children's processing of emotions expressed by peers and adults: an fMRI study. Social Neuroscience, 5(56), 543-559.

Hoffman, M. L. (1987). The contribution of empathy to justice and moral judgment. In N. Eisenberg \& J. Strayer (Eds.), Cambridge studies in social and emotional development. Empathy and its development (p. 47-80). New York, NY: Cambridge University Press.

Holmbeck, G. N., Johnson, S. Z., Wills, K. E., McKernon, W., Rose, B., Erklin, S., \& Kemper, T. (2002). Observed and perceived parental overprotection in relation to psychosocial adjustment in preadolescents with a physical disability: The mediational role of behavioral autonomy. Journal of Consulting and Clinical Psychology, 70(1), 96-110.

Horsley, T. A., de Castro, B. O., \& Van der Schoot, M. (2010). In the eye of the beholder: eyetracking assessment of social information processing in aggressive behavior. Journal of Abnormal Child Psychology, 38(5), 587-599.

Hosie, J. A., Gray, C. D., Russell, P. A., Scott, C., \& Hunter, N. (1998). The matching of facial expressions by deaf and hearing children and their production and comprehension of emotion labels. Motivation and Emotion, 22(4), 293-313. 
Hunnius, S., de Wit, T. C., Vrins, S., \& von Hofsten, C. (2011). Facing threat: infants' and adults' visual scanning of faces with neutral, happy, sad, angry, and fearful emotional expressions. Cognition and Emotion, 25(2), 193-205.

Jack, R. E., Blais, C., Scheepers, C., Schyns, P. G., \& Caldara, R. (2009). Cultural confusions show that facial expressions are not universal. Current Biology, 19(18), 1543-1548.

Jack, R. E., Garrod, O. G., Yu, H., Caldara, R., \& Schyns, P. G. (2012). Facial expressions of emotion are not culturally universal. Proceedings of the National Academy of Sciences of the United States of America, 109(19), 7241-7244.

Jessen, S., Altvater-Mackensen, N., \& Grossmann, T. (2016). Pupillary responses reveal infants' discrimination of facial emotions independent of conscious perception. Cognition, 150, 163-169.

Joormann, J., Waugh, C. E., \& Gotlib, I. H. (2015). Cognitive bias modification for interpretation in Major Depression: Effects on memory and stress reactivity. Clinical Psychological Science, 3(1), 126-139.

Kret, M. E., \& de Gelder, B. (2013). When a smile becomes a fist: the perception of facial and bodily expressions of emotion in violent offenders. Experimental Brain Research, 228(4), 399-410.

Kret, M. E., Denollet, J., Grezes, J., \& de Gelder, B. (2011). The role of negative affectivity and social inhibition in perceiving social threat: an fMRI study. Neuropsychologia, 49(5), 1187-1193.

Kret, M. E., Roelofs, K., Stekelenburg, J. J., \& de Gelder, B. (2013). Emotional signals from faces, bodies and scenes influence observers' face expressions, fixations and pupilsize. Frontiers in Human Neuroscience, 7, 1-9.

Kret, M. E., \& Sjak-Shie, E. E. (2019). Preprocessing pupil size data: Guidelines and code. Behavior Research Methods, 51(3), 1336-1342.

Laeng, B., Saether, L., Holmlund, T., Wang, C. E., Waterloo, K., Eisemann, M., \& Halvorsen, M. (2013). Invisible emotional expressions influence social judgments and pupillary responses of both depressed and non-depressed individuals. Frontiers in Psychology, 4, 291.

Lee, K. U., Kim, J., Yeon, B., Kim, S. H., \& Chae, J. H. (2013). Development and standardization of Extended ChaeLee Korean Facial Expressions of Emotions. Psychiatry Investigation, 10(2), 155-163.

Letourneau, S. M., \& Mitchell, T. V. (2011). Gaze patterns during identity and emotion judgments in hearing adults and deaf users of American Sign Language. Perception, 40(5), 563-575.

Little, R. J. A. (1988). A test of missing completely at random for multivariate data with missing values. Journal of the American Statistical Association, 83, 1198-1202.

Liversedge, S. P., \& Findlay, J. M. (2000). Saccadic eye movements and cognition. Trends in Cognitive Sciences, 4(1), 6-14.

Lundqvist, D., \& Ohman, A. (2005). Emotion regulates attention: The relation between facial configurations, facial emotion, and visual attention. Visual Cognition, 12(1), 51-84.

Ma, D. S., Correll, J., \& Wittenbrink, B. (2015). The Chicago face database: A free stimulus set of faces and norming data. Behavior Research Methods, 47(4), 1122-1135.

Macchi Cassia, V., Pisacane, A., \& Gava, L. (2012). No own-age bias in 3-year-old children: more evidence for the role of early experience in building face-processing biases. Journal of Experimental Child Psychology, 113(3), 372-382.

Marusak, H. A., Carre, J. M., \& Thomason, M. E. (2013). The stimuli drive the response: an fMRI study of youth processing adult or child emotional face stimuli. Neuroimage, 83, 679-689.

McClure, E. B. (2000). A meta-analytic review of sex differences in facial expression 
processing and their development in infants, children, and adolescents. Psychological Bulletin, 126, 424-453.

Meaux, E., Hernandez, N., Carteau-Martin, I., Martineau, J., Barthelemy, C., BonnetBrilhault, F., \& Batty, M. (2014). Event-related potential and eye tracking evidence of the developmental dynamics of face processing. European Journal of Neuroscience, 39(8), 1349-1362.

Meuwissen, A. S., Anderson, J. E., \& Zelazo, P. D. (2017). The creation and validation of the Developmental Emotional Faces Stimulus Set. Behavior Research Methods, 49(3), 960-966.

Mitchell, R., \& Karchmer, M. (2004). Chasing the mythical ten percent: Parental hearing status of deaf and hard of hearing students in the United States. Sign Language Studies, 4(2), 138-163.

Miller, G. A., \& Chapman, J. P. (2001). Misunderstanding analysis of covariance. Journal of Abnormal Psychology, 110, 40-48.

Naruse, S., Hashimoto, T., Mori, K., Tsuda, Y., Takahara, M., \& Kagami, S. (2013). Developmental changes in facial expression recognition in Japanese school-age children. Journal of Medical Investigation, 60, 114-120.

Netten, A. P., Rieffe, C., Theunissen, S. C., Soede, W., Dirks, E., Briaire, J. J., \& Frijns, J. H. (2015). Low empathy in deaf and hard of hearing (pre)adolescents compared to normal hearing controls. PLoS ONE, 10(4), e0124102.

Norbury, C. F., Gooch, D., Wray, C., Baird, G., Charman, T., Simonoff, E., . . Pickles, A. (2016). The impact of nonverbal ability on prevalence and clinical presentation of language disorder: evidence from a population study. Journal of Child Psychology and Psychiatry, 57(11), 1247-1257.

Park, J. Y., Oh, J. M., Kim, S. Y., Lee, M., Lee, C., Kim, B. R., . . An, S. K. (2011). Korean Facial Expressions of Emotion (KOFEE). Yonsei University College of Medicine, Seoul, South Korea.

Partala, T., \& Surakka, V. (2003). Pupil size variation as an indication of affective processing. International Journal of Human-Computer Studies, 59(1-2), 185-198.

Pinquart, M. (2013). Do the parent-child relationship and parenting behaviors differ between families with a child with and without chronic illness? A meta-analysis. Journal of Pediatric Psychology, 38(7), 708-721.

Proksch, J., \& Bavelier, D. (2002). Changes in the spatial distribution of visual attention after early deafness. Journal of Cognitive Neuroscience, 14(5), 687-701.

Raudenbush, S. W., \& Liu, X. (2000). Statistical power and optimal design for multisite randomized trials. Psychological Methods, 5, 199-213.

Rhodes, M. G., \& Anastasi, J. S. (2012). The own-age bias in face recognition: a metaanalytic and theoretical review. Psychological Bulletin, 138(1), 146-174.

Rieffe, C., Broekhof, E., Eichengreen, A., Kouwenberg, M., Veiga, G., da Silva, B. M. S., . . . Frijns, J. H. M. (2018). Friendship and emotion control in pre-adolescents with or without hearing loss. Journal of Deaf Studies and Deaf Education, 23(3), 209-218.

Rieffe, C., Netten, A. P., Broekhof, E., \& Veiga, E. (2015). The role of the environment in children's emotion socialization. In H. Knoors \& M. Marschark (Eds.), Educating Deaf Learners: Creating a Global Evidence Base (pp. 369-388). New York, NY: Oxford University Press.

Russell, J. A., \& Steinberg, R. J. (1994). Is there universal recognition of emotion from facial expression? A review of the cross-cultural studies. Psychological Bulletin, 115(1), 102-141.

Saarni, C. (1999). The development of emotional competence. New York, NY: Guilford Press. Scherer, K. R. (2000). Emotion. In M. Hewstone \& W. Stroebe (Eds.), Introduction to social 
psychology: A European perspective (pp. 151-191). Oxford: Blackwell.

Schurgin, M. W., Nelson, J., Iida, S., Ohira, H., Chiao, J. Y., \& Franconeri, S. L. (2014). Eye movements during emotion recognition in faces. Journal of Vision, 14(13), 1-16.

Sjak-Shie, E. E. (2019). PhysioData Toolbox. Retrieved from https://PhysioDataToolbox.leidenuniv.nl.

Slater, A., \& Quinn, P. C. (2001). Face recognition in the newborn infant. Infant and Child Development, 10(1-2), 21-24.

Stevenson, J., Kreppner, J., Pimperton, H., Worsfold, S., \& Kennedy, C. (2015). Emotional and behavioural difficulties in children and adolescents with hearing impairment: A systematic review and meta-analysis. European Child and Adolescent Psychiatry, 24(5), 477-496.

Theunissen, S. C. P. M., Rieffe, C., Soede, W., Briaire, J. J., Ketelaar, L., Kouwenberg, M., \& Frijns, J. H. M. (2015). Symptoms of psychopathology in hearing-impaired children. Ear and Hearing, 36(4), e190-e198.

Tomblin, J. B., Records, N. L., Buckwalter, P., Zhang, X., Smith, E., \& O’Brien, M. (1997). Prevalence of Specific Language Impairment in kindergarten children. Journal of Speech, Language, and Hearing Research, 40(6), 1245-1260.

Tottenham, N., Borscheid, A., Ellertsen, K., Marcus, D., \& Nelson, C. A. (2002). The NimStim Face Set. Retrieved from: http://www.macbrain.org/faces/index.htm.

Tottenham, N., Tanaka, J. W., Leon, A. C., McCarry, T., Nurse, M., Hare, T. A., . . Nelson, C. (2009). The NimStim set of facial expressions: judgments from untrained research participants. Psychiatry Research, 168(3), 242-249.

Trentacosta, C. J., \& Fine, S. E. (2010). Emotion Knowledge, Social Competence, and Behavior Problems in Childhood and Adolescence: A Meta-Analytic Review. Social Development, 19(1), 1-29.

van Eldik, T., Treffers, P. D. A., Veerman, J. W., \& Verhulst, F. C. (2004). Mental health problems of Deaf dutch children as indicated by parents' responses to the Child Behavior Checklist. American Annals of the Deaf, 148(5), 390-395.

Wagner, H. L. (1993). On measuring performance in category judgment studies of nonverbal behaviors. Journal of Nonverbal Behavior, 17, 3-28.

Waltzman, S. B. (2006). Cochlear implants: current status. Expert Review of Medical Devices, 3, 647-655.

Wang, Y., Su, Y., Fang, P., \& Zhou, Q. (2011). Facial expression recognition: Can preschoolers with cochlear implants and hearing aids catch it? Research in Developmental Disabililities, 32(6), 2583-2588.

Wang, Y., Su, Y., \& Yan, S. (2016). Facial expression recognition in children with cochlear implants and hearing aids. Frontiers in Psychology, 7, 1-6.

Wang, Y., Zhou, W., Cheng, Y., \& Bian, X. (2017). Gaze patterns in auditory-visual perception of emotion by children with hearing aids and hearing children. Frontiers in Psychology, 8, 1-9.

Watanabe, K., Matsuda, T., Nishioka, T., \& Namatame, M. (2011). Eye gaze during observation of static faces in deaf people. PLOS ONE, 6(2), e16919.

Wechsler, D. (1989). Manual for the Wechsler Preschool and Primary Scale of IntelligenceRevised. San Antonio, TX: The Psychological Corporation.

Wechsler, D. (1991). Manual for the Wechsler Intelligence Scale for Children-Third Edition. San Antonio, TX: The Psychological Corporation.

White, L. K., Suway, J. G., Pine, D. S., Bar-Haim, Y., \& Fox, N. A. (2011). Cascading effects: the influence of attention bias to threat on the interpretation of ambiguous information. Behaviour Research and Therapy, 49(4), 244-251.

WHO. (2018, April 2). Fact sheets: Autism spectrum disorders. Retrieved from 
https://www.who.int/news-room/fact-sheets/detail/autism-spectrum-disorders.

WHO. (2019, March 20). Fact sheets: Deafness and hearing loss. Retrieved from https://www.who.int/news-room/fact-sheets/detail/deafness-and-hearing-loss.

Widen, S. C. (2013). Children's interpretation of facial expressions: The long path from valence-based to specific discrete categories. Emotion Review, 5(1), 72-77.

Wiefferink, C. H., Rieffe, C., Ketelaar, L., De Raeve, L., \& Frijns, J. H. (2013). Emotion understanding in deaf children with a cochlear implant. Journal of Deaf Studies and Deaf Education, 18(2), 175-186.

Ziv, M., Most, T., \& Cohen, S. (2013). Understanding of emotions and false beliefs among hearing children versus deaf children. Journal of Deaf Studies and Deaf Education, 18(2), 161-174. 


\section{Table 1.}

Characteristics of the participants.

\begin{tabular}{lll}
\hline Characteristic & DHH & TH \\
\hline$N$ & 55 & 72 \\
Age, years, mean $(S D)$ & $6.45(2.10)$ & $6.03(1.77)$ \\
Gender, $n(\%)$ & & \\
$\quad$ Male & $27(49 \%)$ & $30(42 \%)$ \\
$\quad$ Female & $28(51 \%)$ & $42(58 \%)$ \\
Nonverbal intelligence, mean $(S D)^{\mathrm{a}}$ & $9.49(2.71)$ & $10.35(2.57)$ \\
Parental education level, mean $(S D)^{\mathrm{b}}$ & $3.32(1.04)$ & $3.59(0.96)$ \\
Type of hearing device, $n(\%)$ & & \\
Cochlear implant & $50(92 \%)$ & \\
Hearing aid & $5(8 \%)$ & \\
Degree of hearing loss, $n(\%)$ & & \\
Mild & $2(4 \%)$ & \\
Moderate & $2(4 \%)$ & \\
Severe to profound & $51(92 \%)$ & \\
Age at intervention, years, mean $(S D)$ & $2.54(1.32)$ & \\
Duration of using hearing device, years, mean $(S D)$ & $3.91(1.91)$ & \\
\hline
\end{tabular}

Note: $\mathrm{DHH}=$ deaf and hard of hearing; $\mathrm{TH}=$ typically hearing.

${ }^{a}$ For nonverbal intelligence, age-corrected norm scores are presented. The grand population mean is 10 and the standard deviation is 3 .

bParental education level: $1=$ no/primary education; 2 = lower general secondary education; $3=$ higher general secondary education; 4 = college/university. 
Table 2.

Fixed and random effects in the generalized linear mixed models of gaze patterns and physiological arousal.

\begin{tabular}{|c|c|c|c|c|c|c|c|c|c|c|c|c|}
\hline \multirow[b]{3}{*}{ Fixed/random effect } & \multicolumn{4}{|c|}{ Fixation ratios within AOIs } & \multicolumn{4}{|c|}{ Fixation ratios within entire faces } & \multicolumn{4}{|c|}{ Physiological arousal (pupil dilation) } \\
\hline & \multicolumn{2}{|c|}{ Predefined category } & \multicolumn{2}{|c|}{ Interpreted category } & \multicolumn{2}{|c|}{ Predefined category } & \multicolumn{2}{|c|}{ Interpreted category } & \multicolumn{2}{|c|}{ Predefined category } & \multicolumn{2}{|c|}{ Interpreted category } \\
\hline & $\operatorname{Coef}(\delta)$ & $95 \% \mathrm{CI}$ & $\operatorname{Coef}(\delta)$ & $95 \% \mathrm{CI}$ & Coef $(\delta)$ & $95 \% \mathrm{CI}$ & $\operatorname{Coef}(\delta)$ & $95 \% \mathrm{CI}$ & $\operatorname{Coef}(\delta)$ & $95 \% \mathrm{CI}$ & $\operatorname{Coef}(\delta)$ & $95 \% \mathrm{CI}$ \\
\hline Intercept & .18 & {$[.17, .20]$} & .19 & {$[.18, .20]$} & .96 & {$[.96, .97]$} & .97 & {$[.97, .98]$} & .13 & {$[.09, .17]$} & .14 & {$[.11, .16]$} \\
\hline Age & $n s$ & & $n s$ & & $n s$ & & $n s$ & & $n s$ & & $n s$ & \\
\hline Group & $n s$ & & $n s$ & & $n s$ & & $n s$ & & $.03(.08)$ & {$[-.03, .09]$} & $n s$ & \\
\hline Category(AN) & $-.00(.02)$ & {$[-.02, .01]$} & $n s$ & & $.01(.10)$ & {$[.003, .02]$} & $n s$ & & $.01(.03)$ & {$[-.03, .05]$} & $n s$ & \\
\hline Category(FE) & $.01(.05)$ & {$[.00, .02]$} & $n s$ & & $.01(.12)$ & {$[.005, .02]$} & $n s$ & & $-.01(.04)$ & {$[-.06, .03]$} & $n s$ & \\
\hline Category(HA) & $.01(.02)$ & {$[-.01, .02]$} & $n s$ & & $.01(.08)$ & {$[.000, .02]$} & $n s$ & & $.03(.08)$ & {$[-.01, .07]$} & $n s$ & \\
\hline AOI(Eyes) & $.27(1.08)$ & {$[.26, .28]$} & $.27(1.08)$ & {$[.26, .28]$} & -- & & -- & & -- & & -- & \\
\hline AOI(Nose) & $-.07(.27)$ & {$[-.08,-.06]$} & $-.07(.27)$ & {$[-.08,-.06]$} & -- & & -- & & -- & & -- & \\
\hline Category(AN) x Group & $n s$ & & $n s$ & & $n s$ & & $n s$ & & $-.00(.01)$ & {$[-.07, .06]$} & $n s$ & \\
\hline Category(FE) x Group & $n s$ & & $n s$ & & $n s$ & & $n s$ & & $-.02(.04)$ & {$[-.08, .05]$} & $n s$ & \\
\hline Category(HA) x Group & $n s$ & & $n s$ & & ns & & $n s$ & & $-.09(.23)$ & {$[-.15,-.02]$} & $n s$ & \\
\hline AOI x Group & $n s$ & & $n s$ & & -- & & -- & & -- & & -- & \\
\hline Variance(Intercept) & .002 & {$[.002, .003]$} & .002 & {$[.002, .003]$} & .001 & {$[.001, .001]$} & .001 & {$[.001, .001]$} & .014 & {$[.01, .02]$} & .014 & {$[.01, .02]$} \\
\hline Residual & .062 & {$[.060, .063]$} & .062 & {$[.060, .063]$} & .013 & {$[.012, .013]$} & .013 & {$[.012, .013]$} & .132 & {$[.13, .14]$} & .133 & {$[.13, .14]$} \\
\hline$N$ included & 15183 & & 15183 & & 5061 & & 5061 & & 55082 & & 55082 & \\
\hline
\end{tabular}

Note: Group was coded as $-1=\mathrm{DHH}, 1=\mathrm{TH}$ (reference). Category (i.e., predefined/interpreted emotion category) was coded as $-2=$ anger $($ AN), $-1=$ fear $(\mathrm{FE}), 1=$

happiness (HA), $2=$ neutral emotion (reference). Area of interest (AOI) was coded as $-1=$ eyes, $0=$ nose, $1=$ mouth (reference). An " $n s$ " indicates that the variable was removed from the final model due to insignificance. A "-." indicates that the variable was not included in the full model. Significant values $(p<0.05)$ are bolded. Coef $=$ unstandardized coefficient; $\mathrm{CI}=$ confidence interval. $\delta=$ standardized effect size, calculated by dividing fixed coefficient by the square root of the sum of Level 1 (residual) and Level 2 (intercept) variances (formula suggested by Raudenbush and Liu, 2000). $N$ included = number of cases included in the analysis. 


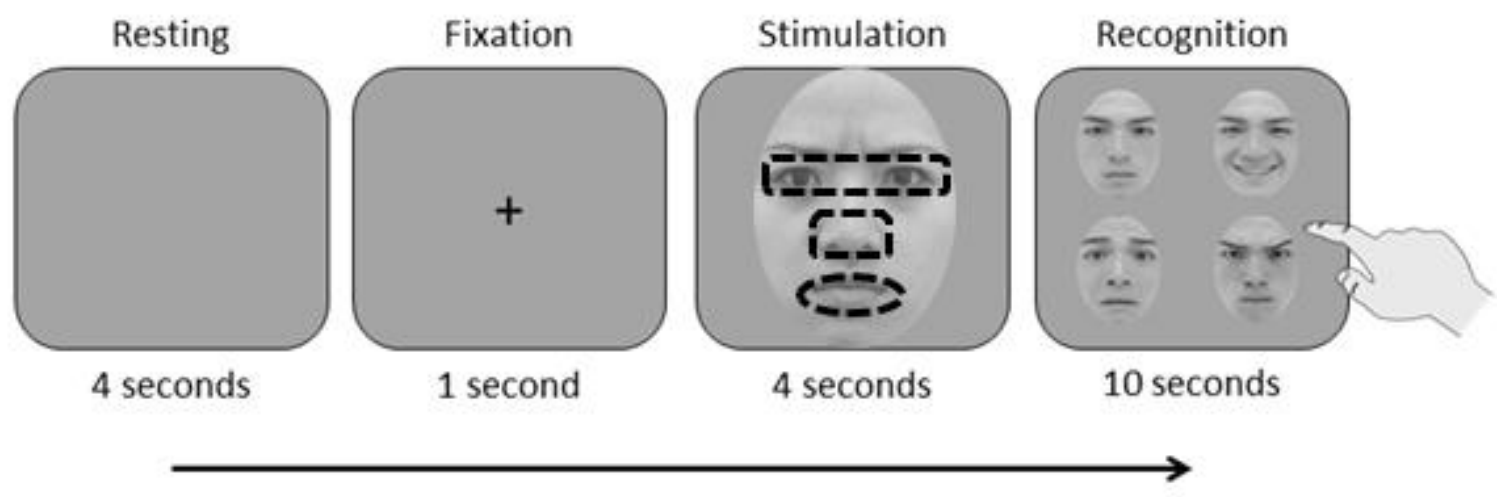

Figure 1. An example trial sequence. The dashed lines on the face in the stimulation phase denote the three areas of interest (AOIs) used in the analyses, which were not presented to the participants during the experiment. The face images shown here were downloaded from http://bml.ym.edu.tw/tfeid/modules/wfdownloads/ with the owner's permission for using the face images of these two models in scientific publications. 
A.

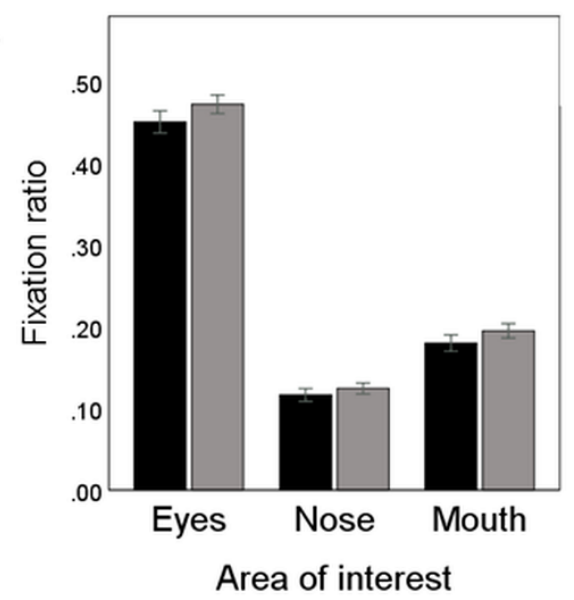

C.

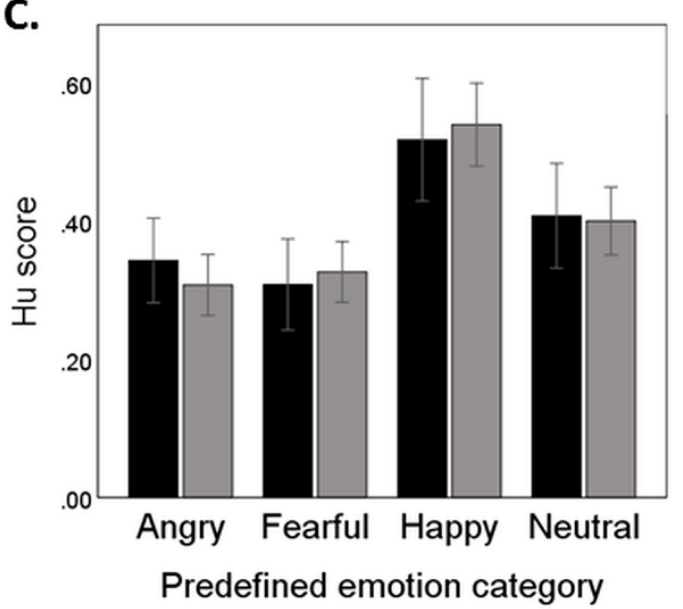

Predefined emotion category

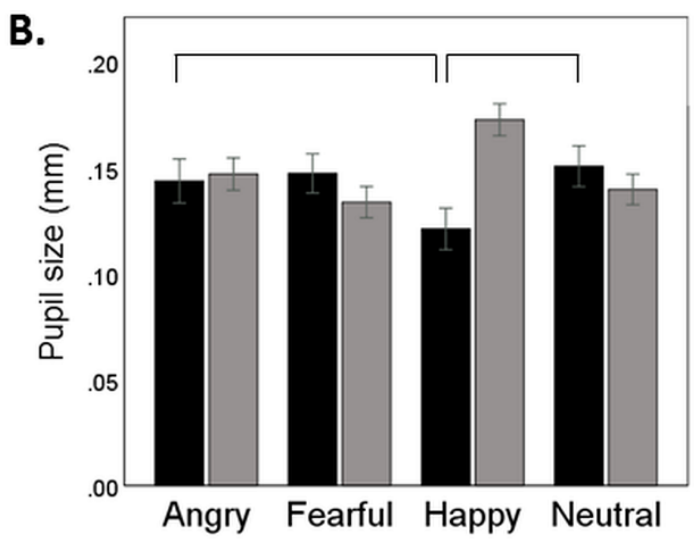

Predefined emotion category

D.

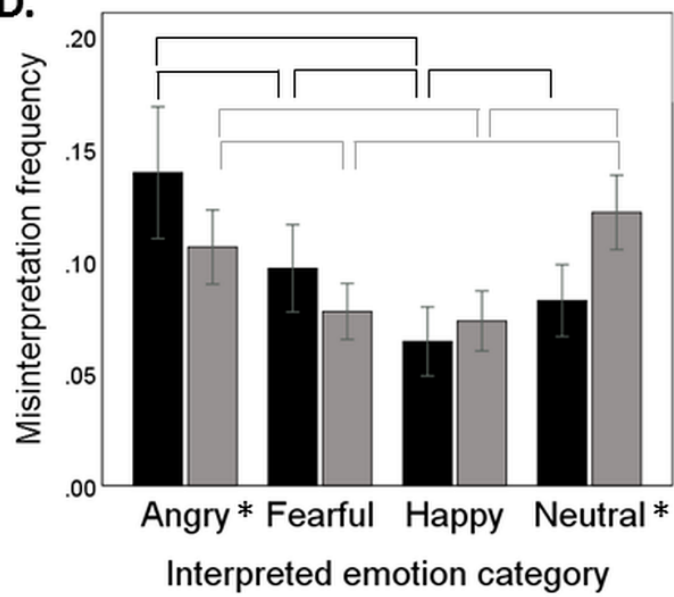

Typically hearing $(\mathrm{TH})$

Figure 2. Graphic representations of the interaction effects with group membership. When reading emotional faces, (A) all children, $\mathrm{DHH}$ and $\mathrm{TH}$, looked longer at eyes than at nose and mouth; (B) DHH children were less aroused by happy faces than by angry and neutral faces, while TH children were similarly aroused by all faces; (C) all children, DHH and TH, performed better on happy faces than on angry, fearful, and neutral faces; (D) DHH children more often misinterpreted a face as non-happy than as happy, while TH children more often misinterpreted a face as angry or neutral than as fearful or happy. The error bars represent $95 \%$ confidence interval. The black lines represent significant differences between emotion categories within the DHH group. The grey lines represent significant differences between emotion categories within the TH group. *significant between-group difference $(p<.05)$. 
Author Note:

We thank all the children and their parents who participated in this study. We also thank Dr. C. M. Wu, Ms. C. J. Lin, and Ms. Y. Y. Lin at Chang-Gung Memorial Hospital for their help in recruiting participants, Ms. E. Chen from Tobii Pro and SOLO at Leiden University for their technical support, and Ms. J. Schoerke for her suggestions and corrections on the language in this manuscript. This work was supported by The Royal Netherlands Academy of Arts and Sciences (Project number 530-5CDP17), and the Taiwan Ministry of Education (Project number GSSA1071007013). The dataset and associated information used in this study are shared publicly on the Leiden University archiving platform DataverseNL (https://doi.org/10.34894/0GWR9W). 